\title{
WEAKLY COMPACT POSITIVE OPERATORS ON SUMMABLE FUNCTIONS
}

\author{
PAUL C. SHIELDS
}

ABSTRACT. This paper uses elementary integration and operator techniques combined with some theorems of Nakano to give a simple proof of the well-known Dunford-Pettis theorem for positive operators.

The Dunford-Pettis theorem [1] states that a weakly compact linear operator on $L^{1}$ which has separable range maps weakly convergent sequences into norm convergent sequences. We prove

THEOREM 1. If $T$ is a weakly compact positive linear operator on $L^{1}(S, \Sigma, \mu)$ then $T$ maps weakly convergent sequences into norm convergent sequences.

For simplicity let us assume that $\mu$ is a finite measure. The space $L^{1}(S, \Sigma, \mu)$ is a vector lattice where $f \geqq 0$ is to mean that $f(s) \geqq 0$ a.e. The norm bounded operators on $L^{1}(S, \Sigma, \mu)$ also form a vector lattice where $T \geqq 0$ means that $T f \geqq 0$ if $f \geqq 0$ and $T \vee S$ is defined for $f \geqq 0$ by

$$
(T \vee S) f=\sup _{0 \leqq g \leqq f} T g+S(f-g) .
$$

Let $B\left(L^{1}\right)$ denote the set of order-continuous operators on $L^{1}$, that is, $T \in B\left(L^{1}\right)$ if and only if $T$ maps dominated a.e. convergent sequences into dominated a.e. convergent sequences. It is fairly easy to see that $B\left(L^{1}\right)$ consists of all the norm-bounded operators on $L^{1}$. Except for this latter result, all the above definitions and results hold for $L^{\infty}$. If $L^{\infty}$ is not finite-dimensional then $B\left(L^{\infty}\right)$ is a proper lattice subspace of the set of all norm-bounded operators on $L^{\infty}$.

Let $L$ denote either of the two spaces $L^{1}$ or $L^{\infty}$ and for $N \subseteq B(L)$; let

$$
N^{\perp}=\{T|| T|\wedge| C \mid=0 \text { for all } C \in N\} \text {. }
$$

Suppose $\varphi$ is the operator defined by $\varphi f=\int f d \mu, f \in L$, and that $F$ is the set of operators in $B(L)$ of finite rank. It is easy to see that

(i) $T \in F^{\perp \perp}$ if and only if $|T|=\sup _{n}|T| \wedge n \varphi$.

Received by the editors March 16, 1971.

AMS (MOS) subject classifications (1970). Primary 47B55, 46A40.

Key words and phrases. Weakly compact operator, positive operator, vector lattice.

(c) American Mathematical Society 1973 
Nakano [3, Theorem 5.2] gives the following characterization of $F^{\perp \perp}$.

(ii) $T \in F^{\perp \perp}$ if and only if $T$ maps order-bounded sequences which converge in measure into order-bounded sequences which converge a.e.

A second result of Nakano [3, Theorem 5.3] is the following:

(iii) If $T \in B(L)$ is dominated by an operator in $F$, then $T$ maps weakly convergent sequences into dominated sequences which converge a.e.

It will now be shown that Theorem 1 is a consequence of (iii). To do this put

$$
\lambda(A)=T^{*}\left(\chi_{A}\right), \quad A \in \Sigma,
$$

where $T^{*}$ is the adjoint of the given positive weakly compact operator $T$ on $L^{1}(S, \Sigma, \mu)$. Since $T^{* *}$ maps $L^{1 * *}$ into $L^{1}$ it follows that $\lambda$ is weakly countably additive and hence countably additive and absolutely continuous with respect to $\mu$ [2, Theorem IV. 10.1]. Thus we can write

and obtain

$$
T^{*} f=\int f d \lambda, \quad f \in L^{\infty},
$$

(iv) If $0 \leqq f_{n} \leqq f$ and $\left\{f_{n}\right\}$ converges in measure to 0 , then $\left\{T^{*} f_{n}\right\}$ converges to 0 a.e.

The proof of (iv) is quite similar to the analogous scalar measure result. For any given $\varepsilon>0$, put $E_{n}=\left\{s \mid f_{n}(s) \geqq \varepsilon\right\}$ so that

$$
\begin{aligned}
T^{*} f_{n} & =\int_{E_{n}} f_{n} d \lambda+\int_{E_{n}^{c}} f_{n} d \lambda \\
& \leqq \lambda\left(E_{n}\right)\|f\|_{\infty}+\varepsilon \lambda(S) .
\end{aligned}
$$

Since $\lambda$ is absolutely continuous with respect to $\mu$ it follows that $\left\|\lambda\left(E_{n}\right)\right\|_{\infty} \rightarrow 0$ and hence that $\lim \lambda\left(E_{n}\right)=0$ a.e.

Now apply the result (ii) to $T^{*}$ to conclude that $T^{*} \in F^{\perp \perp}$ and hence that $T^{*}=\sup _{n}\left(T^{*}\right) \wedge n \varphi$. From this it follows easily that $T=\sup _{n} T \wedge n \varphi$.

This establishes the result

(v) If $T$ is a weakly compact positive operator on $L^{1}$, then there is a sequence $\left\{T_{n}\right\}$ of positive operators which increases to $T$ such that each $T_{n}$ is dominated by an operator of finite rank.

Suppose $\left\{a_{m}\right\}$ converges to 0 weakly. Then $T a_{m}=T_{n} a_{m}+\left(T-T_{n}\right) a_{m}$ so that

$$
\int\left|T a_{m}\right| d \mu \leqq \int\left|T_{n} a_{m}\right| d \mu+\int\left(T-T_{n}\right)\left|a_{m}\right| d \mu .
$$

Note that $\left\{\left|a_{m}\right|\right\}$ is weakly compact, while $\left\{\left(T^{*}-T_{n}^{*}\right) 1\right\}$ decreases to 0 , so that

$$
\lim _{n} \int\left(T-T_{n}\right)\left|a_{m}\right| d \mu=0
$$


uniformly in $m$. Furthermore, if $n$ is fixed, the result (iii) gives

$$
\lim _{m} \int\left|T_{n} a_{m}\right| d \mu=0 .
$$

These two facts combine with (vi) to show that $\lim _{m} \int\left|T a_{m}\right| d \mu=0$, which completes the proof of Theorem 1 .

REMARK. If one can show that

$$
|T|^{* *}=\left|T^{* *}\right| \text { for } T \in B\left(L^{1}\right),
$$

it is easy to remove the assumption that $T$ be positive in Theorem 1 . The result (vii), however, seems very difficult to prove.

\section{BIBLIOGRAPHY}

1. N. Dunford and B. J. Pettis, Linear operations on summable functions, Trans. Amer. Math. Soc. 47 (1940), 323-392. MR 1, 338.

2. N. Dunford and J. T. Schwartz, Linear operators. I: General theory, Pure and Appl. Math., vol. 7, Interscience, New York, 1958. MR 22 \#8302.

3. H. Nakano, Product spaces of semi-ordered linear spaces, J. Fac. Sci. Hokkaido Univ. Ser. I 12 (1953), 163-210. MR 16, 49.

Current address: Department of Mathematics, Stanford University, Stanford, California 94305 